\title{
Performance Analysis of CDMA-based Networks with Interference Cancellation, for Batched Poisson Traffic Under the Bandwidth Reservation Policy
}

\author{
Ioannis D. Moscholios ${ }^{1}$, Vassilios G. Vassilakis ${ }^{2}$, Georgios A. Kallos ${ }^{3}$ and Michael D. Logothetis ${ }^{4}$ \\ 1. Dept. of Informatics \& Telecommunications, University of Peloponnese, 22100 Tripolis, Greece. \\ 2. Computer Laboratory, University of Cambridge, CB3 OFD Cambridge, U.K. \\ 3. BT Technology, Service and Operations, IP5 3RE Ipswich, U.K. \\ 4. WCL, Dept. of Electrical \& Computer Engineering, University of Patras, 26504 Patras, Greece. \\ Emails: 1dm@uop.gr, vv274@cl.cam.ac.uk, george.kallos@bt.com, mlogo@upatras.gr
}

\begin{abstract}
CDMA-based technologies deserve assiduous analysis and evaluation. We study the performance, at call-level, of a CDMA cell with interference cancellation capabilities, while assuming that the cell accommodates different service-classes of batched Poisson arriving calls. The partial batch blocking discipline is applied for Call Admission Control (CAC). To guarantee certain Quality of Service (QoS) for each service-class, the Bandwidth Reservation (BR) policy is incorporated in the CAC; i.e., a fraction of system resources is reserved for highspeed service-classes. We propose a new multirate loss model for the calculation of time and call congestion probabilities. The notion of local (soft) and hard blocking, users activity, interference cancellation, as well as the BR policy, are incorporated in the model. Although the steady state probabilities of the system do not have a product form solution, time and call congestion probabilities can be efficiently determined via approximate but recursive formulas. Simulation verified the high accuracy of the new formulas. We also show the consistency of the proposed model in respect of its parameters, while comparison of the proposed model with that of Poisson input shows its necessity.
\end{abstract}

Keywords-CDMA; time-call congestion; Batched Poisson process; recursive formula; bandwidth reservation.

\section{INTRODUCTION}

The CDMA (Code-Division Multiple Access) belongs to a family of channel access techniques used by various radio communication technologies [1]. As an example: a) the Wideband CDMA (W-CDMA) and the Time Division CDMA are used in $3 \mathrm{G}$ mobile technology standards (UMTS, CDMA2000) and b) the Multi-Carrier CDMA and the Large Area Synchronised CDMA have been considered in $4 \mathrm{G}$ networks [2], [3]. Recently, there started some research efforts in envisioning CDMA technology as a key multiple access method in $5 \mathrm{G}$ networks [4]. While nothing is clear yet with regard to $5 \mathrm{G}$ networks, it's likely that they will integrate different existing and future wireless network technologies (including CDMA-based) to ensure seamless roaming between various technology standards. Thus, it is evident that CDMA systems deserve assiduous analysis and evaluation.

We consider a CDMA reference cell of fixed capacity consisting of an integer number of channels, and study its performance in the uplink. The cell accommodates calls from
1 service-classes, which require an integer number of channels. A new call is accepted in the cell if the requested channels are available. Specifically, the admission of a new call is based on the increase of the total interference (own-cell and other-cell interference plus thermal noise) caused by the new call's acceptance. An accepted call remains in the system for an exponentially distributed service time. Due to interferences, a new call may be blocked in any system state (soft or local blocking), if its acceptance increases the noise of all in-service calls above a tolerable level. This admission policy corresponds to the Complete Sharing (CS) policy in wired networks, whereby all calls compete for all bandwidth resources [5].

The CS policy has been adopted in [6]-[13], in which, the recursive formulas proposed for the Call Blocking Probabilities (CBP) calculation, resemble the KaufmanRoberts recursion [14], [15]. The latter refers to a link that accommodates Poisson arriving calls of $K$ service-classes with different bandwidth requirements and generally distributed service times; we name this model Erlang Multirate Loss Model (EMLM). In [7], [9] and [13], calls follow a Poisson process, while the models of [6], [10] cover Poisson, Bernoulli and Pascal traffic, based on the model of [16]. In [8], [11] and [12] calls come from a finite number of users. Apart from the different arrival processes considered in [6]-[13], these models also differ in the modelling of local blocking; e.g., the modelling approach of [6], [10] is more complicated compared to [7], as it is shown in [11]. Therefore, herein, we adopt the model of [7] for the local blocking.

In this paper, we assume that new calls follow a batched Poisson process while the batch size is generally distributed. An arriving batch can be partially accepted in the cell due to lack of resources. In that case, some calls of the batch will be serviced and the rest will be blocked. This discipline is named Partial Batch Blocking (PBB). In addition, we study the effect of the Bandwidth Reservation (BR) policy in Time and Call Congestion probabilities (TC and CC probabilities, respectively) [17]. These probabilities coincide for Poisson arrivals (PASTA property [17]). If the arrival process is batched Poisson (with PBB), CC probabilities are higher than TC probabilities. The latter highly differ between different service-classes, under the CS policy. To achieve equalization of TC probabilities, we apply the BR policy. This is a sine qua 
non policy in the case that multiple rate calls share a system, and it has been broadly applied (e.g., [18]-[20]). Moreover, we study the impact of Interference Cancellation (IC) on TC and $\mathrm{CC}$ probabilities. IC receivers reduce the own-cell interference (e.g., [21]), a fact that decreases TC and CC probabilities in the proposed model. Due to local (soft) blocking and the BR policy, the proposed model does not have a product form solution. However, we propose approximate but recursive formulas for the efficient TC and CC probabilities calculation. Simulation verifies the high accuracy of the analytical results.

A call-level analysis in CDMA networks assuming a batched Poisson process and the existence of the BR policy has not been considered before. On the other hand, many papers study this call arrival process in wired networks (see e.g., [22]-[26]). In [22], the EMLM is extended to include a batched Poisson process under the geometric batch size distribution and the PBB discipline. In [23], the batch size is generally distributed. Herein, we name the model of [23], Batch Poisson EMLM (BP-EMLM). In [24], the BP-EMLM is extended to include the BR policy, while in [25], to include the case where in-service calls can compress their bandwidth during their lifetime in the system. In [26], the arrival process remains batched Poisson but in-service calls behave according to an ON-OFF model.

This paper is organized as follows: In Section II, we present the basic relations in the uplink direction of a CDMA reference cell. In subsection II.A, we review the relations for the total received power of a service-class $k$ call with or without the IC existence. In subsection II.B, we determine the max. number of service-class $k$ calls in the cell under the IC presence. In subsection II.C, we calculate the uplink capacity and the bandwidth requirement of service-class $k$ calls. In Section III, we consider Poisson arrivals and calculate CBP when the system operates with hard blocking only (subsection III.A), and when the system operates both with hard and soft blocking (subsection III.B). In Section IV, we consider batched Poisson arrivals and calculate $\mathrm{TC}$ and $\mathrm{CC}$ probabilities under hard blocking only (subsection IV.A), as well as under both hard and soft blocking (subsection IV.B). In both subsections III.B and IV.B, we also consider the case of IC and the application of the BR policy. In Section V, we present analytical and simulation results in order to evaluate the new formulas. We conclude in Section VI.

\section{BASIC RELATIONS IN THE UPLINK OF A CDMA CELL}

A. Determination of the total received power of a serviceclass $k$ call

Consider a CDMA reference cell controlled by a base station and surrounded by other cells. We examine the uplink direction and model the cell as a loss system, which accommodates $K$ different service-classes. A service-class $k$ call $(k=1, \ldots, K)$ alternates between transmission (active) periods and non-transmission (passive) periods. The ratio of "active" over "active + passive" periods is the activity factor of a service-class $k$ call, $v_{k}$. Typical values of $v_{k}$ are: $v_{k}=1.0$ or $v_{k}$ $=0.67$ if $k$ is a data or a voice service-class, respectively.

In CDMA systems, a single user "sees" the signals generated by other users as interference. In that case, the base station's capacity in the CDMA reference cell is limited by the Multiple Access Interference (MAI) [1]. The latter consists of: 1) the own-cell interference, $P_{\text {own }}$, caused by users of the reference cell and 2) the other-cell interference, $P_{\text {other }}$, which refers to the interference power received from users of neighbouring cells. Since MAI has a stochastic nature, we refer to the soft capacity of the radio interface [27]. We also consider thermal noise, $P_{\text {noise }}$, which expresses the interference of an empty CDMA system. A typical value of the thermal noise power density, in W-CDMA systems, is $174 \mathrm{dBm} / \mathrm{Hz}$ [1].

The values of $P_{\text {own }}$ can be reduced by the IC. The IC efficiency, $\beta$, is defined by the ratio [21]:

$\beta=\frac{P_{o w n}^{N O I C}-P_{o w n}}{P_{o w n}^{N O I C}} \Rightarrow P_{o w n}=P_{o w n}^{N O I C}(1-\beta)$

where $P_{o w n}^{N O I C}$ is the own-cell interference without IC.

For $\beta$, we assume that is: i) constant $(0 \leq \beta<1)$, ii) common to all calls, and iii) independent of the radio link conditions and the implementation of the receiver ([21], [28]).

Due to the IC existence and by denoting as $p_{k}$ the total received power from a service-class $k$ user, we write the power control equation for service-class $k$ as follows [21]:

$$
\left(\frac{E_{b}}{N_{0}}\right)_{k}=G_{k} \frac{p_{k}}{\left(P_{o w n}-p_{k}\right)(1-\beta)+P_{o t h e r}+P_{n o i s e}}
$$

where $\left(E_{b} / N_{0}\right)_{k}$ is the signal energy per bit divided by the noise spectral density, required to meet a predefined Block Error Rate, $G_{k}=\frac{W}{v_{k} R_{k}}$ is the processing gain of service-class $k$ in the uplink direction with user activity factor $v_{k}$, data rate $R_{k}$ and $W$ the chip rate of $3840 \mathrm{kcps}$ (in W-CDMA systems).

Based on (2), the values of $p_{k}$ can be obtained by:

$$
p_{k}=\left(\left(P_{\text {own }}(1-\beta)+P_{\text {other }}+P_{\text {noise }}\right) /\left(1-\beta+G_{k} /\left(\frac{E_{b}}{N_{0}}\right)_{k}\right)\right)
$$

Note 1: If $\beta=0$ then (2), (3) result in [1]:

$$
\begin{gathered}
\left(\frac{E_{b}}{N_{0}}\right)_{k}=G_{k} \frac{p_{k}}{P_{\text {total }}-p_{k}} \\
p_{k}=\frac{P_{\text {total }}}{1+G_{k} /\left(E_{b} / N_{0}\right)_{k}}
\end{gathered}
$$

where $P_{\text {total }}=P_{\text {own }}+P_{\text {other }}+P_{\text {noise }}$ is the total received power at the base station.

\section{B. Calculation of the max. number of service-class $k$ calls in the cell}

Let $N_{k}$ be the max. number of service-class $k$ calls in the cell. If $P_{\text {own }}=p_{k} N_{k}$, then $P_{\text {own }}$ can be written as: 


$$
P_{\text {own }}=\frac{N_{k}\left(P_{\text {other }}+P_{\text {noise }}\right)}{(1-\beta)-N_{k}(1-\beta)+G_{k} /\left(E_{b} / N_{0}\right)_{k}}
$$

Consider now the Noise Rise, $N R$, expressed as [23]:

$$
N R=P_{\text {total }} / P_{\text {noise }}=\left(P_{\text {own }}+P_{\text {other }}+P_{\text {noise }}\right) / P_{\text {noise }}
$$

The $N R$ is related to the total uplink cell load, $\eta_{U L}$, via [1]:

$$
N R=\frac{1}{1-\eta_{U L}}, \eta_{U L}=\frac{P_{\text {own }}+P_{\text {other }}}{P_{\text {total }}}
$$

Based on (7) and (8) we have:

$$
\frac{P_{\text {own }}+P_{\text {other }}+P_{\text {noise }}}{P_{\text {noise }}}=\frac{1}{1-\eta_{U L}}
$$

Substituting (6) in (9) and solving for $N_{k}$ we have:

$$
N_{k}=\left[(1-\beta)+G_{k} /\left(E_{b} / N_{0}\right)_{k}\right] \frac{\left[\eta_{U L}(\delta+1)-\delta\right]}{\left[1-\beta\left(\eta_{U L}(\delta+1)-\delta\right)\right]}
$$

where $\delta=P_{\text {other }} / P_{\text {noise }}$.

If $\beta=0$ and $\delta=0$ (i.e., $P_{\text {other }}=0$ ), then (10) takes the form:

$$
N_{k}=\left[1+G_{k} /\left(E_{b} / N_{0}\right)_{k}\right] \eta_{U L}
$$

Note 2: If we have perfect power control and the same $\left(E_{b} / N_{0}\right), R, v$ and consequently $G$ and $p$ for all serviceclasses then (10) becomes:

$$
N=\left[(1-\beta)+\frac{G}{\left(E_{b} / N_{0}\right)}\right] \frac{\left[\eta_{U L}(\delta+1)-\delta\right]}{\left[1-\beta\left(\eta_{U L}(\delta+1)-\delta\right)\right]}
$$

where $N$ is the total number of users in the reference cell. Now:

1) if $\beta=0$ then (12) becomes:

$$
N=\left[1+\frac{G}{\left(E_{b} / N_{0}\right)}\right]\left[\eta_{U L}(\delta+1)-\delta\right]
$$

Equation (13) is the same with (8.12) of [1], if:

$$
\eta_{U L}(\delta+1)-\delta=\frac{n_{U L}}{i+1}, i=P_{\text {other }} / P_{o w n}
$$

2) if $\beta>0$, (12) gives the same results with (8) of [21].

\section{Determination of the uplink capacity and the bandwidth} requirement of service-class $k$ calls

Having determined $N_{k}$ via (10), we calculate the spread data rate $R_{s, k}$ of service-class $k$, as:

$$
R_{s, k}=W / N_{k}
$$

Now, we transform the chip rate $W$ to the uplink capacity $C$, and the spread data rate $R_{s, k}$ to the corresponding bandwidth requirement per call, $b_{k}$, of each service-class $k$. To achieve this, we define a basic bandwidth unit ( $b b u)$, which is determined as the greatest common divisor of the required call resources of all service-classes, or it can take an arbitrarily chosen small value. E.g., if $b b u=20 \mathrm{Kcps}$, then $C$ and $b_{k}$ are:

$C=\left\lceil\frac{W}{b b u}\right\rceil=192$ channels, $b_{k}=\left\lceil\frac{R_{s, k}}{b b u}\right\rceil$ channels

\section{Call Blocking Probabilities Assuming Poisson ARRIVALS}

\section{A. A system with hard blocking only}

In connection-oriented systems, every system state $j(j=0$, $1, \ldots, C)$, that shows the occupied channels in the system, can be a blocking or a non-blocking state for service-class $k$ calls, depending on the bandwidth requirement $b_{k}$. Assuming Poisson arrivals, generally distributed service times and the CS policy, then the system is modelled by the EMLM and $q(j)$ 's are given by the Kaufman-Roberts formula [14], [15]:

$$
q(j)= \begin{cases}1 & , \text { for } j=0 \\ \frac{1}{j} \sum_{k=1}^{K} a_{k} b_{k} q\left(j-b_{k}\right), & \text { for } j=1, \ldots, C \\ 0 \quad, & \text { otherwise }\end{cases}
$$

where $\alpha_{k}=\lambda_{k} / \mu_{k}$ is the offered traffic-load of service-class $k$ (in erl) while $\lambda_{k}$ is the mean arrival rate of service-class $k$ calls.

The calculation of TC probabilities of service-class $k, P_{b_{k}}$, is based on the following formula:

$P_{b_{k}}=\sum_{j=C-b_{k}+1}^{C} G^{-1} q(j)$

where $G=\sum_{j=0}^{C} q(j)$ is the normalization constant.

\section{B. A system with both hard blocking and soft blocking}

Two types of states $j$ are considered in CDMA networks, for service-class $k$ calls: a) those that are blocking states (hard blocking states) and b) those that are blocking states with a probability $0<L_{j, k}<1$ (soft or local blocking states) due to the stochastic nature of the other-cell interference. In what follows, we show how to incorporate local blocking in (17).

To consider the other-cell interference in the reference cell, we approximate it by an independent, lognormally distributed random variable with parameters $\mu$ and $\sigma$, given by:

$\mu=\frac{P_{\text {other }}+P_{\text {noise }}}{P_{\text {own }}+P_{\text {other }}+P_{\text {noise }}} C \Rightarrow \mu=\frac{i+i / \delta}{1+i+i / \delta} C, \sigma=\mu$ 
The parameter $\mu=\sigma$ according to the literature (e.g., [6]-[7], [29]-[30]).

If $P_{\text {noise }}$ is not considered, then (19) takes the form:

$\mu=\frac{P_{\text {other }}}{P_{\text {own }}+P_{\text {other }}} C \Rightarrow \mu=\frac{i}{1+i} C, \sigma=\mu$

The local blocking probability in state $j$, denoted as $L_{j}$, is the probability that the other-cell interference is greater than the available cell's capacity $\left(C-t_{k}-j\right)$ and is independent of the bandwidth requirement of service-class $k$ calls:

$L_{j}=1-P\left(j^{\prime}<C-t_{k}-j\right)=1-C D F\left(C-t_{k}-j\right)$

where $j^{\prime}$ denotes the occupied channels due to the other cell interference, $t_{k}$ is the BR parameter that shows the channels reserved for all calls apart from service-class $k$ and $C D F(x)$ is the cumulative distribution function of the lognormal random variable, $x$, determined by:

$C D F(x)=\frac{1}{2}\left(1+\operatorname{erf}\left(\frac{\ln (x)-M}{S \sqrt{2}}\right)\right)$

where erf is the error function, while $M$ and $S$ refer to the parameters of the normal distribution and are given by:

$M=\ln \left(\mu^{2} / \sqrt{\mu^{2}+\sigma^{2}}\right), \quad S=\sqrt{\ln \left(1+\left(\sigma^{2} / \mu^{2}\right)\right)}$

Due to the BR policy, a service-class $k$ call cannot enter the states $j=C-t_{k}+1, \ldots, C$. Consider now a new service-class $k$ call which requires $b_{k}$ channels and has a BR parameter $t_{k}$. The call will be accepted in the cell if all $b_{k}$ channels are assigned to the call simultaneously. This means, that the other-cell interference remains the same during the allocation process of these $b_{k}$ channels. We express the passage factor $1-L_{j, b_{k}}$, i.e., the probability that the call is not blocked due to the other-cell interference as a function of $j$ and $b_{k}$ :

$1-L_{j, b_{k}}=1-L_{j+b_{k}-1}=C D F\left(C-t_{k}-j-b_{k}+1\right)$

Due to $(24)$, the transition rate from state $\left(j-b_{k}\right)$ to state $j$, equals $\left(1-L_{j-b_{k}, b_{k}}\right) \lambda_{k}=\left(1-L_{j-1}\right) \lambda_{k}$. To determine $q(j)$, we propose the following formula:

$q(j)=\left\{\begin{array}{l}1 \quad, \quad \text { for } j=0 \\ \frac{1}{j} \sum_{k=1}^{K} a_{k} D_{k}\left(j-b_{k}\right) q\left(j-b_{k}\right)\left(1-L_{j-b_{k}, b_{k}}\right), \text { for } j=1, \ldots, C \\ 0 \quad, \text { otherwise }\end{array}\right.$
$D_{k}\left(j-b_{k}\right)= \begin{cases}b_{k} \text { for } j \leq C-t_{k} \\ 0 & \text { for } j>C-t_{k}\end{cases}$ where the values of $\left(1-L_{j-b_{k}, b_{k}}\right)$ are given by (24).

In the case of the CS policy, (25) takes the form [7]:

$$
q(j)= \begin{cases}1 & , \text { for } j=0 \\ \frac{1}{j} \sum_{k=1}^{K} a_{k} b_{k} q\left(j-b_{k}\right)\left(1-L_{j-b_{k}, b_{k}}\right), \text { for } j=1, \ldots, C \\ 0 \quad, \text { otherwise }\end{cases}
$$

The determination of TC (and CC) probabilities of serviceclass $k, P_{b_{k}}$, is based on the formula:

$P_{b_{k}}=\sum_{j=0}^{C} G^{-1} L_{j, j+b_{k}} q(j)$

where $G=\sum_{j=0}^{C} q(j)$ and $L_{j, j+b_{k}}=1-C D F\left(C-t_{k}-j-b_{k}+1\right)$.

\section{Congestion Probabilities Assuming Batched POISSON ARRIVALS}

\section{A. A system with hard blocking only}

Consider a system of capacity $C$ channels that accommodates $K$ service-classes. Calls of service-class $k$ $(k=1, \ldots, K)$ follow a batched Poisson process with arrival rate $\lambda_{k}$ and batch size distribution $B_{m}^{(k)}$, where $B_{m}^{(k)}$ is the probability that a new batch contains $m$ calls of service-class $k$. Each service-class $k$ call requires $b_{k}$ channels and has an exponentially distributed service time with mean $\mu_{k}^{-1}$. Assuming the PBB discipline, the BP-EMLM results, whereby $q(j)$ 's are given by the following accurate formula [23]:

$$
q(j)= \begin{cases}1 & \text { for } j=0 \\ \frac{1}{j} \sum_{k=1}^{K} \alpha_{k} b_{k} \sum_{l=1}^{\left\lfloor j / b_{k}\right\rfloor} \hat{B}_{l-1}^{(k)} q\left(j-l b_{k}\right) & \text { for } j=1, \ldots, C \\ 0 & \text { otherwise }\end{cases}
$$

where $\hat{B}_{l}^{(k)}=\sum_{m=l+1}^{\infty} B_{m}^{(k)}$.

If $B_{m}^{(k)}=1$ for $m=1$ and $B_{m}^{(k)}=0$ for $m>1$ we have the Poisson process. In that case, $q(j)$ 's are given by (17).

If service-class $k$ calls arrive in batches of size $s_{k}$ where $s_{k}$ is geometrically distributed with parameter $\gamma_{k}$, i.e., $\operatorname{Pr}\left(s_{k}=r\right)=\left(1-\gamma_{k}\right) \gamma_{k}^{r-1}, r \geq 1$, then the model of [23] coincides with that of [16] and (29) becomes:

$q(j)= \begin{cases}1 & \text { for } j=0 \\ \frac{1}{j} \sum_{k=1}^{K} \alpha_{k} b_{k} \sum_{l=1}^{\left\lfloor j / b_{k}\right\rfloor} \gamma_{k}^{l-1} q\left(j-l b_{k}\right) & \text { for } j=1, \ldots, C \\ 0 & \text { otherwise }\end{cases}$ 
Based on (29), we can calculate:

a) The average number of service-class $k$ calls when the system state is $j, y_{k}(j)$ :

$y_{k}(j)=\alpha_{k} \sum_{l=1}^{\left\lfloor j / b_{k}\right\rfloor} \hat{B}_{l-1}^{(k)} q\left(j-l b_{k}\right) / q(j)$

b) The average number of in-service service-class $k$ calls, $\bar{n}_{k}$ :

$$
\bar{n}_{k}=\sum_{j=1}^{C} G^{-1} y_{k}(j) q(j)
$$

c) The CC probabilities of service-class $k$ calls, $C_{b_{k}}$ :

$$
C_{b_{k}}=\left(\alpha_{k} \hat{B}_{k}-\bar{n}_{k}\right) / \alpha_{k} \hat{B}_{k}
$$

where $\hat{B}_{k}=\sum_{m=1}^{\infty} m B_{m}^{(k)}$.

In the case of the geometric batch size distribution with parameter $\gamma_{k}$ we have: $\hat{B}_{k}=\frac{1}{1-\gamma_{k}}$.

d) The TC probabilities of service-class $k$ calls, $P_{b_{k}}$, via (18).

\section{B. A system with both hard blocking and soft blocking}

To introduce local blocking in the BP-EMLM of [23] we adopt [7]. In that case, the transition rate from state $\left(j-l b_{k}\right)$ to state $j$, equals $\left(1-L_{j-b_{k}, b_{k}}\right) \lambda_{k} \hat{B}_{l-1}^{(k)}=\left(1-L_{j-1}\right) \lambda_{k} \hat{B}_{l-1}^{(k)}$, where $l=1$, $2, \ldots,\left\lfloor j / b_{k}\right\rfloor$ (see also [31], if only the CS policy is applied).

To calculate $q(j)$ in a CDMA cell which accommodates batched Poisson calls of $K$ service-classes under the PBB discipline and the BR policy, we propose the formula:

$$
q(j)=\left\{\begin{array}{lc}
1 & \text { for } j=0 \\
\frac{1}{j} \sum_{k=1}^{K} \alpha_{k} D_{k}\left(j-b_{k}\right) \sum_{l=1}^{\left\lfloor j / b_{k}\right\rfloor} \hat{B}_{l-1}^{k)} q\left(j-l b_{k}\right)\left(1-L_{j-b_{k}, b_{k}}\right) & \text { for } j=1, \ldots, C \\
0 & \text { otherwise }
\end{array}\right.
$$

where: $\hat{B}_{l}^{(k)}=\sum_{m=l+1}^{\infty} B_{m}^{(k)},\left(1-L_{j-b_{k}, b_{k}}\right)=\left(1-L_{j-1}\right)$.

Note that if $B_{m}^{(k)}=1$ for $m=1$ and $B_{m}^{(k)}=0$ for $m>1$ then the arrival process is Poisson and $q(j)$ 's are given by (25).

In the case of the geometric batch distribution with parameter $\gamma_{k},(34)$ becomes:

$$
q(j)=\left\{\begin{array}{lc}
1 & \text { for } j=0 \\
\frac{1}{j} \sum_{k=1}^{K} \alpha_{k} D_{k}\left(j-b_{k}\right) \sum_{l=1}^{\left\lfloor j / b_{k}\right\rfloor} \gamma_{k}^{l-1} q\left(j-l b_{k}\right)\left(1-L_{j-b_{k}, b_{k}}\right) \text { for } j=1, \ldots, C \\
0 & \text { otherwise }
\end{array}\right.
$$

Based on (34), we calculate:

a) The average number of service-class $k$ calls when the system state is $j \leq C-t_{k}, y_{k}(j)$ : $y_{k}(j)=\alpha_{k} \sum_{l=1}^{\left\lfloor j / b_{k}\right\rfloor} \hat{B}_{l-1}^{(k)} q\left(j-l b_{k}\right)\left(1-L_{j-b_{k}, b_{k}}\right) / q(j)$

b) The values of $\bar{n}_{k}$, via (32)

c) The CC probabilities of service-class $k$ calls, $C_{b_{b}}$, via (33). Note that $C_{b_{k}}$ refers to the CC probability of a call of a batch.

d) The TC probabilities of service-class $k$ calls, $P_{b_{k}}$, via (28).

\section{NUMERICAL RESULTS}

Consider a W-CDMA cell with IC capabilities of the efficiency levels: $\beta=0.0, \beta=0.3$, and $\beta=0.7$. In the cell, $K=3$ service-classes of batched Poisson traffic are accommodated, under the BR policy. We obtain analytical results of TC and $\mathrm{CC}$ probabilities through the proposed model, and compare them with the corresponding simulation results. We also compare them with analytical results when the input traffic is Poisson. Simulations are carried out via the SIMSCRIPT III language [32], and the obtained results are mean values of 7 runs. Confidence intervals of $95 \%$ for the mean values are very small and are not presented. Both $\mathrm{TC}$ and $\mathrm{CC}$ probabilities simulation results are very close to the corresponding analytical results; thus, we only present analytical results, in figures. However, in order to show that the analytical and simulation results are very close, we comparatively present them in tables.

Table I contains the traffic characteristics of the serviceclasses. As far as the batch size distribution is concerned, it is geometrically distributed with $\gamma_{1}=0.833, \gamma_{2}=0.750$ and $\gamma_{3}=$ 0.600 , for each corresponding service-class. The call service time is exponentially distributed with mean $\mu_{1}^{-1}=\mu_{2}^{-1}=\mu_{3}^{-1}=1$. In addition, let: $\eta_{U L}=0.75, i=0.35$, $\delta=2.0, b b u=13.5 \mathrm{kcps}$.

When $\beta=0.0$, then: $b_{1}=4, b_{2}=7, b_{3}=19$ and $t_{1}=15$, $t_{2}=12, t_{3}=0$. Observe that the BR parameters are chosen according to the TC probabilities equalization rule, i.e., $b_{1}+t_{1}$ $=b_{2}+t_{2}=b_{3}+t_{3}$. Similarly, when $\beta=0.3$ then $b_{1}=4, b_{2}=6, b_{3}$ $=18$ and $t_{1}=14, t_{2}=12, t_{3}=0$, while when $\beta=0.7$ then $b_{1}=4, b_{2}$ $=6, b_{3}=16$ and $t_{1}=12, t_{2}=10, t_{3}=0$. In the x-axis of Figs 1-6 and in the $1^{\text {st }}$ column of Tables I-VII, the offered traffic load of the $1^{\text {st }}, 2^{\text {nd }}$ and $3^{\text {rd }}$ service-class increases in steps of $0.2,0.1$ and 0.05 erl, respectively. So, point 1 refers to: $\left(\alpha_{1}, \alpha_{2}, \alpha_{3}\right)=$ $(0.2,0.1,0.05)$ while point 8 to: $\left(\alpha_{1}, \alpha_{2}, \alpha_{3}\right)=(1.6,0.8,0.4)$.

TABLE I. Traffic parameters of the three service-classes.
\begin{tabular}{|c|c|c|c|c|c|c|}
\hline & & & & & \\
\hline $\begin{array}{c}\text { Service- } \\
\text { class } k\end{array}$ & $\begin{array}{c}R_{k} \\
(\mathrm{kbps})\end{array}$ & $v_{k}$ & $\left(\frac{E_{b}}{N_{0}}\right)_{k}$ & $\left(\frac{E_{b}}{N_{0}}\right)_{k}$ & $\gamma_{k}$ & $\begin{array}{c}a_{k} \\
\text { (in erl) } \\
(\mathrm{in} \mathrm{dB})\end{array}$ \\
\hline 1 & 7.95 & 0.67 & 4.0 & 2.51 & 0.833 & 0.2 \\
\hline 2 & 12.20 & 0.67 & 4.0 & 2.51 & 0.750 & 0.1 \\
\hline 3 & 32.00 & 1.0 & 3.0 & 2.00 & 0.600 & 0.05 \\
\hline
\end{tabular}

Figures 1-3 present the analytical TC probabilities of each service-class, while Figs. 4-6 present the corresponding CC probabilities. Based on these results, we observe/confirm that: 1) $\mathrm{CC}$ probabilities are higher than $\mathrm{TC}$ probabilities in the case of the batched Poisson process. This result is anticipated and shows the consistency of the proposed model. 
2) The increase of $\beta$ results in the decrease of TC and CC probabilities. This result is also expected and shows again the consistency of the proposed model since the IC reduces the own-cell interference.

3) The TC and CC probabilities obtained when the arrival process is Poisson fail to approximate the corresponding TC and $\mathrm{CC}$ probabilities in the case of the batched Poisson process (either with or without the BR policy). This result shows the necessity of the proposed model; it was also anticipated, since a batched Poisson process is more "peaked" and "bursty" than a Poisson process.

4) The application of the BR policy increases the TC and CC probabilities of the $1^{\text {st }}$ and $2^{\text {nd }}$ service-class and slightly decreases the corresponding probabilities of the $3^{\text {rd }}$ serviceclass. In general, the BR policy increases substantially the blocking probabilities of low-speed calls, while the simultaneous decrease of blocking probabilities of high-speed calls is not always noticeable.

In Tables II-III, we present the analytical and simulation TC probabilities, for $\beta=0.0$ and 0.7 , when the BR policy is applied. In the $2^{\text {nd }}$ column of these tables we consider the analytical and equalized TC probabilities, named $P_{b, \text { equal. }}$. In

Tables IV-V, we present the analytical and simulation CC probabilities results, when the BR policy is not applied, for the last call of a service-class $k$ batch $(k=1,2,3)$ and $\beta=0.0$ and 0.7 , respectively. The analytical calculation of this $\mathrm{CC}$ probability is given by $C_{b_{k, \text { last }}}=\left(1-\gamma_{k}\right) C_{b_{k}}$. The corresponding analytical and simulation $\mathrm{CC}$ probabilities results, when the BR policy is applied, are presented in Tables VI-VII. As Tables II-VII reveal, the simulation results are very close to the analytical results. Of similar accuracy are all the simulation results which correspond to the analytical results of Figs. 1-6. Furthermore, we see that the increase of $\beta$, decreases the $\mathrm{CC}$ probabilities of the last call of a serviceclass $k$ batch.

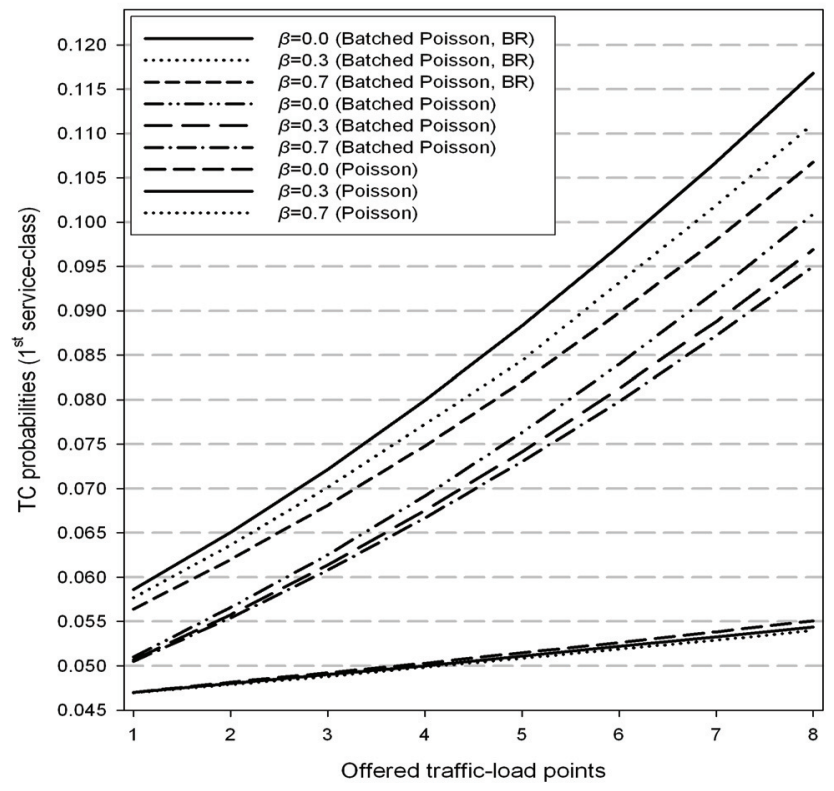

Fig. 3. TC probabilities of the $1^{\text {st }}$ service-class.

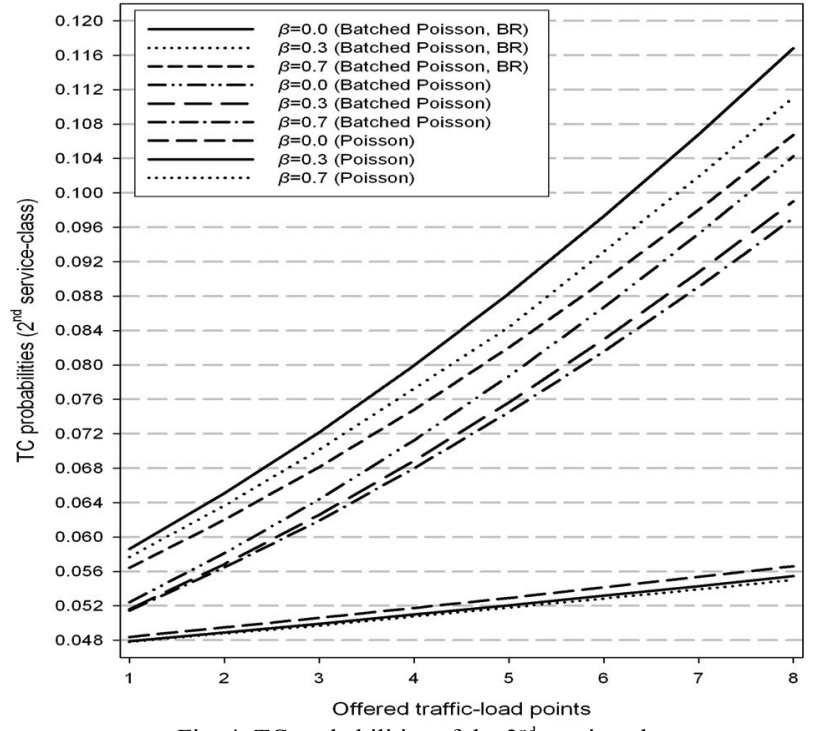

Fig. 4. TC probabilities of the $2^{\text {nd }}$ service-class.

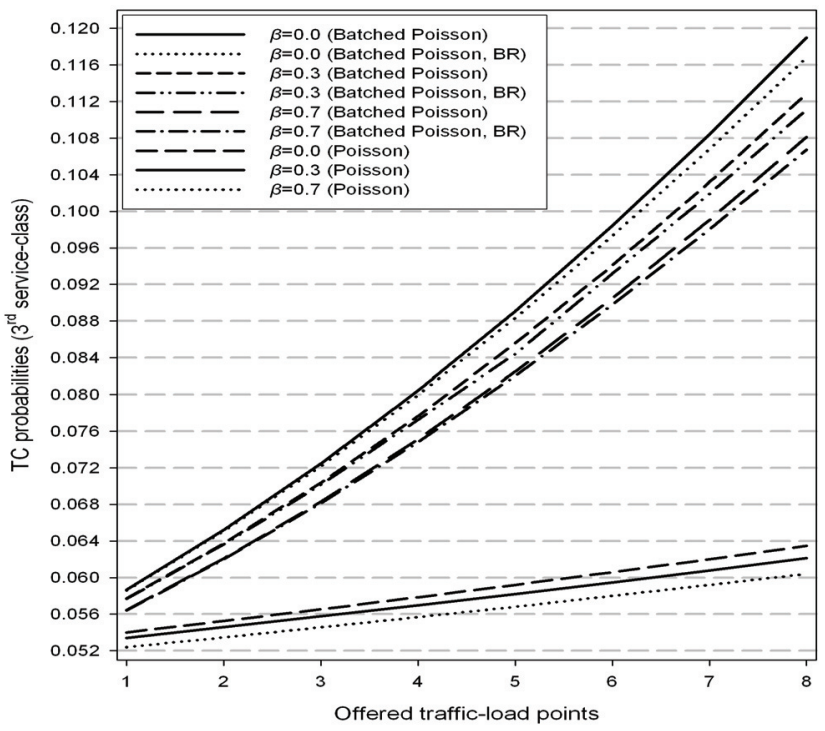

Fig. 5. TC probabilities of the $3^{\text {rd }}$ service-class.

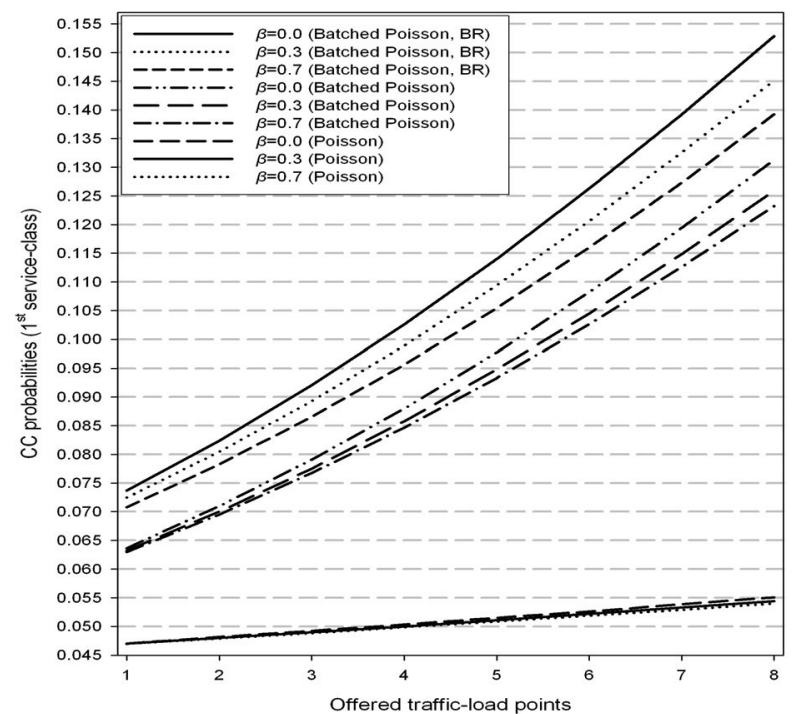

Fig. 6. CC probabilities of the $1^{\text {st }}$ service-class. 


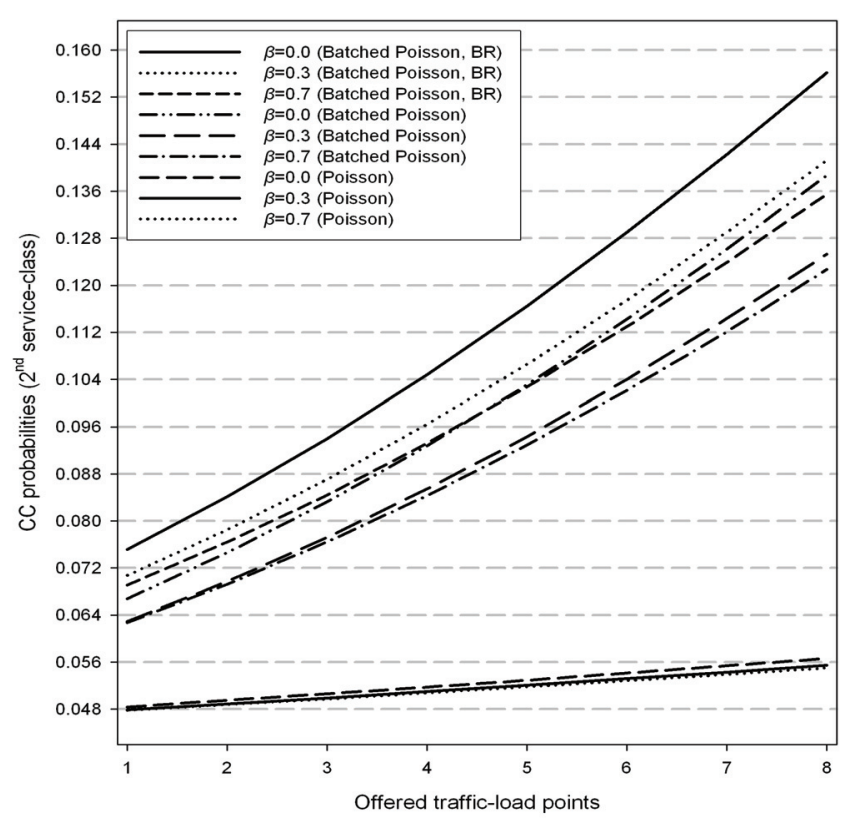

Fig. 7. CC probabilities of the $2^{\text {nd }}$ service-class.

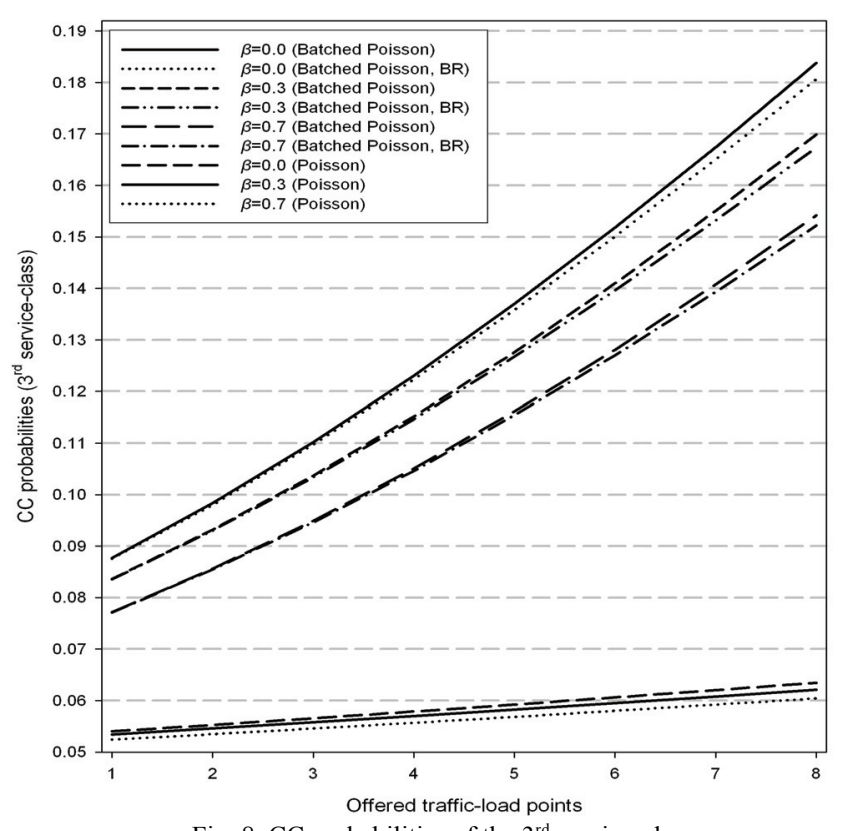

Fig. 8. CC probabilities of the $3^{\text {rd }}$ service-class.

TABLE II. Equalized TC probabilities (when $\beta=0.0$ )

\begin{tabular}{|c|c|c|c|c|}
\hline $\begin{array}{l}\text { Offered } \\
\text { traffic-load } \\
\text { point }\end{array}$ & $\begin{array}{c}P_{b, \text { equal. }} \\
\text { (analyt.) }\end{array}$ & $\begin{array}{c}P_{b_{1}} \\
\text { (sim.) }\end{array}$ & $\begin{array}{c}P_{b_{2}} \\
\text { (sim.) }\end{array}$ & $\begin{array}{c}P_{b_{3}} \\
\text { (sim.) }\end{array}$ \\
\hline 1 & 0.05861 & 0.05864 & 0.05863 & 0.05865 \\
\hline 2 & 0.06507 & 0.06516 & 0.06514 & 0.06517 \\
\hline 3 & 0.07218 & 0.07224 & 0.07225 & 0.07220 \\
\hline 4 & 0.07994 & 0.08009 & 0.08007 & 0.08007 \\
\hline 5 & 0.08833 & 0.08840 & 0.08836 & 0.08842 \\
\hline 6 & 0.09730 & 0.09736 & 0.09737 & 0.09734 \\
\hline 7 & 0.10680 & 0.10690 & 0.10685 & 0.10689 \\
\hline 8 & 0.11679 & 0.11688 & 0.11690 & 0.11685 \\
\hline
\end{tabular}

TABLE III. Equalized TC probabilities (when $\beta=0.7$ )

\begin{tabular}{|c|c|c|c|c|}
\hline $\begin{array}{l}\text { Offered } \\
\text { traffic-load } \\
\text { point }\end{array}$ & $\begin{array}{c}P_{b_{\text {,equal. }}} \\
\text { (analyt.) }\end{array}$ & $\begin{array}{c}P_{b_{1}} \\
\text { (sim.) }\end{array}$ & $\begin{array}{c}P_{b_{2}} \\
\text { (sim.) }\end{array}$ & $\begin{array}{c}P_{b_{3}} \\
\text { (sim.) }\end{array}$ \\
\hline 1 & 0.05641 & 0.05644 & 0.05643 & 0.05647 \\
\hline 2 & 0.06200 & 0.06206 & 0.06204 & 0.06200 \\
\hline 3 & 0.06813 & 0.06819 & 0.06815 & 0.06818 \\
\hline 4 & 0.07480 & 0.07488 & 0.07484 & 0.07486 \\
\hline 5 & 0.08202 & 0.08210 & 0.08213 & 0.08209 \\
\hline 6 & 0.08977 & 0.08980 & 0.08982 & 0.08983 \\
\hline 7 & 0.09802 & 0.09810 & 0.09807 & 0.09806 \\
\hline 8 & 0.10674 & 0.10680 & 0.10677 & 0.10681 \\
\hline
\end{tabular}

TABLE IV. CC probabilities - last call of a batch (when $\beta=0.0$, no BR)

\begin{tabular}{|c|c|c|c|c|c|c|}
\hline $\begin{array}{l}\text { Offered } \\
\text { traffic- } \\
\text { load } \\
\text { point }\end{array}$ & $\begin{array}{c}C_{b_{1, \text { lat }}} \\
\text { (analyt.) }\end{array}$ & $\begin{array}{c}C_{b_{1, \text { lats }}} \\
\text { (sim.) }\end{array}$ & $\begin{array}{c}C_{b_{2, \text { lat }}} \\
\text { (analyt.) }\end{array}$ & $\begin{array}{c}C_{b_{2, \text { lat }}} \\
\text { (sim.) }\end{array}$ & $\begin{array}{c}C_{b_{3, \text { lat }}} \\
\text { (analyt.) }\end{array}$ & $\begin{array}{c}C_{b_{3, \text { last }}} \\
\text { (sim.) }\end{array}$ \\
\hline 1 & 0.01061 & 0.01062 & 0.01669 & 0.01670 & 0.03506 & 0.03509 \\
\hline 2 & 0.01183 & 0.01185 & 0.01864 & 0.01866 & 0.03935 & 0.03939 \\
\hline 3 & 0.01318 & 0.01319 & 0.02081 & 0.02082 & 0.04408 & 0.04410 \\
\hline 4 & 0.01466 & 0.01468 & 0.02319 & 0.02320 & 0.04923 & 0.04927 \\
\hline 5 & 0.01629 & 0.01630 & 0.02577 & 0.02579 & 0.05479 & 0.05483 \\
\hline 6 & 0.01804 & 0.01805 & 0.02856 & 0.02857 & 0.06072 & 0.06077 \\
\hline 7 & 0.01990 & 0.01992 & 0.03153 & 0.03156 & 0.06698 & 0.06702 \\
\hline 8 & 0.02187 & 0.02188 & 0.03467 & 0.03469 & 0.07352 & 0.07356 \\
\hline
\end{tabular}

TABLE V. CC probabilities - last call of a batch (when $\beta=0.7$, no BR)

\begin{tabular}{|c|c|c|c|c|c|c|}
\hline $\begin{array}{l}\text { Offered } \\
\text { traffic- } \\
\text { load } \\
\text { point }\end{array}$ & $\begin{array}{c}C_{b_{1, \text { lat }}} \\
\text { (analyt.) }\end{array}$ & $\begin{array}{l}C_{b_{1, \text { last }}} \\
(\mathrm{sim} .)\end{array}$ & $\begin{array}{c}C_{b_{2, \text { ast }}} \\
\text { (analyt.) }\end{array}$ & $\begin{array}{l}C_{b_{2, \text { last }}} \\
(\mathrm{sim} .)\end{array}$ & $\begin{array}{c}C_{b_{3, \text { lat }}} \\
\text { (analyt.) }\end{array}$ & $\begin{array}{l}C_{b_{3, \text { lat }}} \\
\text { (sim.) }\end{array}$ \\
\hline 1 & 0.01049 & 0.01050 & 0.01568 & 0.01569 & 0.03086 & 0.03090 \\
\hline 2 & 0.01159 & 0.01159 & 0.01731 & 0.01732 & 0.03424 & 0.03429 \\
\hline 3 & 0.01279 & 0.01280 & 0.01910 & 0.01912 & 0.03795 & 0.03800 \\
\hline 4 & 0.01411 & 0.01411 & 0.02109 & 0.02110 & 0.04203 & 0.04206 \\
\hline 5 & 0.01555 & 0.01557 & 0.02322 & 0.02323 & 0.04646 & 0.04651 \\
\hline 6 & 0.01711 & 0.01712 & 0.02554 & 0.02556 & 0.05122 & 0.05126 \\
\hline 7 & 0.01877 & 0.01889 & 0.02802 & 0.02803 & 0.05630 & 0.05634 \\
\hline 8 & 0.02050 & 0.02052 & 0.03067 & 0.03069 & 0.06167 & 0.06170 \\
\hline
\end{tabular}

TABLE VI. CC probabilities - last call of a batch (when $\beta=0.0$, under BR)

\begin{tabular}{|c|c|c|c|c|c|c|}
\hline $\begin{array}{l}\text { Offered } \\
\text { traffic- } \\
\text { load } \\
\text { point }\end{array}$ & $\begin{array}{c}C_{b_{1, \text { lat }}} \\
\text { (analyt.) }\end{array}$ & $\begin{array}{l}C_{b_{1, \text { last }}} \\
\text { (sim.) }\end{array}$ & $\begin{array}{c}C_{b_{2, \text { lata }}} \\
\text { (analyt.) }\end{array}$ & $\begin{array}{l}C_{b_{2, \text { ast }}} \\
\text { (sim.) }\end{array}$ & $\begin{array}{c}C_{b_{3, \text { lata }}} \\
\text { (analyt.) }\end{array}$ & $\begin{array}{l}C_{b_{3, \text { lat }}} \\
(\mathrm{sim} .)\end{array}$ \\
\hline 1 & 0.01228 & 0.01230 & 0.01870 & 0.01873 & 0.03503 & 0.03507 \\
\hline 2 & 0.01374 & 0.01375 & 0.02100 & 0.02103 & 0.03926 & 0.03929 \\
\hline 3 & 0.01534 & 0.01535 & 0.02349 & 0.02351 & 0.04390 & 0.04394 \\
\hline 4 & 0.01710 & 0.01712 & 0.02620 & 0.02621 & 0.04893 & 0.04897 \\
\hline 5 & 0.01900 & 0.01903 & 0.02912 & 0.02914 & 0.05432 & 0.05434 \\
\hline 6 & 0.02104 & 0.02106 & 0.03224 & 0.03227 & 0.06000 & 0.06007 \\
\hline 7 & 0.02320 & 0.02324 & 0.03556 & 0.03558 & 0.06604 & 0.06607 \\
\hline 8 & 0.02547 & 0.02550 & 0.03903 & 0.03905 & 0.07226 & 0.07228 \\
\hline
\end{tabular}


TABLE VII. CC probabilities - last call of a batch (when $\beta=0.7$, under BR)

\begin{tabular}{|c|c|c|c|c|c|c|}
\hline $\begin{array}{l}\text { Offered } \\
\text { traffic- } \\
\text { load } \\
\text { point }\end{array}$ & $\begin{array}{c}C_{b_{1} \text {, ast }} \\
\text { (analyt.) }\end{array}$ & $\begin{array}{c}C_{b_{1, \text { last }}} \\
(\mathrm{sim} .)\end{array}$ & $\begin{array}{c}C_{b_{2, \text { last }}} \\
\text { (analyt.) }\end{array}$ & $\begin{array}{c}C_{b_{2, \text { last }}} \\
(\mathrm{sim} .)\end{array}$ & $\begin{array}{c}C_{b_{3, \text { ast }}} \\
\text { (analyt.) }\end{array}$ & $\begin{array}{c}C_{b_{3, \text { last }}} \\
\text { (sim.) }\end{array}$ \\
\hline 1 & 0.01180 & 0.01182 & 0.01727 & 0.01729 & 0.03084 & 0.03086 \\
\hline 2 & 0.01305 & 0.01306 & 0.01909 & 0.01911 & 0.03418 & 0.03420 \\
\hline 3 & 0.01442 & 0.01443 & 0.02110 & 0.02113 & 0.03785 & 0.03787 \\
\hline 4 & 0.01594 & 0.01596 & 0.02330 & 0.02334 & 0.04180 & 0.04184 \\
\hline 5 & 0.01758 & 0.01761 & 0.02568 & 0.02570 & 0.04618 & 0.04621 \\
\hline 6 & 0.01930 & 0.01933 & 0.02820 & 0.02824 & 0.05081 & 0.05086 \\
\hline 7 & 0.02120 & 0.02122 & 0.03098 & 0.03099 & 0.05573 & 0.05576 \\
\hline 8 & 0.02320 & 0.02322 & 0.03387 & 0.03390 & 0.06089 & 0.06091 \\
\hline
\end{tabular}

\section{CONCLUSION}

We propose a new multirate loss model for the call-level analysis of CDMA networks that support batched Poisson calls from different service-classes. Calls are accepted in the system according to the PBB discipline and the BR policy. The latter benefits high-speed calls in grade-of-service, by reserving a fraction of the available bandwidth. The proposed model takes into account the MAI, the notion of soft and hard blocking and the user's activity, as well as the IC. Due to the existence of soft blocking and the BR policy, our model does not have a product form solution. However, we show an approximate but recursive formula for the efficient calculation of occupancy distribution and, consequently, the determination of $\mathrm{TC}$ and $\mathrm{CC}$ probabilities. In addition, we study the effect of the IC efficiency in these performance measures. Simulation results verify the accuracy of the proposed model.

\section{REFERENCES}

[1] H. Holma and A. Toskala, eds., W-CDMA for UMTS - HSPA Evolution and LTE, $4^{\text {th }}$ edition, Wiley \& Sons, 2007.

[2] U. Varshney, "4G Wireless Networks", IT Professional, IEEE Computer Society, vol. 14, issue 5, pp. 34-39, September/October 2012.

[3] S. Pinter and X. Fernando, "Estimation and equalization of fiberwireless uplink for multiuser CDMA 4G networks", IEEE Trans. Commun., vol. 58, no. 6, pp. 1803-1813, June 2010 .

[4] S. Patel, C. Malhar, K. Kapadiya, "5G: Future Mobile TechnologyVision

2020", Int. Journal of Computer Applications, 54 (17), pp. 6-10, Sept 2012.

[5] K. Ross, Multiservice Loss Models for Broadband Telecommunication Networks, Springer, London, 1995

[6] V. Iversen, V. Benetis, N. Ha, and S. Stepanov, "Evaluation of Multiservice CDMA Networks with Soft Blocking", Proc. ITC Specialist Seminar, pp. 223-227, August/September 2004.

[7] L. Popova and W. Koch, "Analytical performance evaluation of mixed services with variable data rates for the uplink of UMTS", Proc. ISWCS'06, Valencia, Spain, September 2006.

[8] V. Vassilakis, G. Kallos, I. Moscholios and M. Logothetis, "Call-Level Analysis of W-CDMA Networks Supporting Elastic Services of Finite Population", Proc. IEEE ICC 2008, Beijing, China, 19-23 May 2008.

[9] V. Vassilakis, G. Kallos, I. Moscholios and M. Logothetis, "Evaluation of a Call Admission Control Scheme in W-CDMA Cellular Networks", Proc. $6^{\text {th }}$ CSNDSP' 2008, Graz, Austria, 23-25 July 2008.

[10] V. Iversen, "Evaluation of Multi-service CDMA Networks with Soft Blocking", Proc. of $3^{\text {rd }}$ ruSMART 2010 and $10^{\text {th }}$ Int. Conference, NEW2AN 2010, St. Petersburg, Russia, pp. 160-171, 23-25 August 2010.
[11] I. Moscholios, G. Kallos, V. Vassilakis, M. Logothetis and M. Koukias, "Congestion Probabilities in W-CDMA networks supporting calls of finite sources", Proc. of HETNETs 2013, Ilkley, U.K, 11-13 Nov. 2013.

[12] I. Moscholios, M. Katsiva, G. Kallos, V. Vassilakis and M. Logothetis, "Equalization of Congestion Probabilities in a W-CDMA Cell Supporting Calls of Finite Sources With Interference Cancellation", Proc. $9^{\text {th }}$ CSNDSP' 2014, Manchester, U.K, 23-25 July 2014.

[13] V. Vassilakis, I. Moscholios, M. Logothetis and M. Koukias, "A SingleThreshold Model for Handoff Traffic Analysis in Cellular CDMA Networks", Proc. $10^{\text {th }}$ Advanced Int. Conf. on Telecommunications, AICT, Paris, France, 20-24 July 2014.

[14] J. Kaufman, "Blocking in a shared resource environment", IEEE Trans. Commun., 29 (10), pp. 1474-1481, October 1981.

[15] J. Roberts, "A service system with heterogeneous user requirements", in: G. Pujolle (Ed.), Performance of Data Communications systems and their applications, North Holland, Amsterdam, pp.423-431, 1981.

[16] L. Delbrouck, "On the steady state distribution in a service facility with different peakedness factors and capacity requirements", IEEE Trans. Commun., 31 (11), pp. 1209-1211, November 1983.

[17] H. Akimaru and K. Kawashima, Teletraffic - Theory and Applications, $2^{\text {nd }}$ edition, Springer-Verlag, Berlin, 1999.

[18] M. Glabowski, M. Sobieraj, and M. Stasiak, "Modelling limited availability systems with multi-service sources and bandwidth reservation", Proc. $8^{\text {th }}$ AICT, Stuttgart, Germany, June 2012.

[19] I. Moscholios, J. Vardakas, M. Logothetis and M. Koukias, "A Quasirandom Multirate Loss Model supporting Elastic and Adaptive Traffic under the Bandwidth Reservation Policy", Int. Journal on Advances in Networks and Services, 6 (3 \& 4), December 2013, pp. 163-174.

[20] K. Kuppuswamy and D. Lee, "An Analytic Approach to Efficiently Computing Call Blocking Probabilities for Multiclass WDM Networks", IEEE/ACM Trans. Networking, 17 (2), pp. 658-670, April 2009.

[21] C. Rosa, T. Sorensen, J. Wigard and R. Mogensen, "Interference cancellation and 4-branch antenna diversity for W-CDMA uplink packet access", Proc. IEEE VTC 2005, pp. 1758-1762, 30 May - 1 June 2005.

[22] E. van Doorn and F. Panken, "Blocking Probabilities in a Loss System with Arrivals in Geometrically Distributed Batches and Heterogeneous Service Requirements", IEEE/ACM Trans. Networking, 1 (6), pp. 664667, December 1993.

[23] J. Kaufman and K. Rege, "Blocking in a shared resource environment with batched Poisson arrival processes", Performance Evaluation 24, pp. 249-263, February 1996.

[24] I. Moscholios and M. Logothetis, "The Erlang Multirate Loss Model with Batched Poisson Arrival Processes under the Bandwidth Reservation Policy", Computer Communications, 33 (1), pp. S167-S179, Nov. 2010.

[25] I. Moscholios, J. Vardakas, M. Logothetis and A. Boucouvalas, "Congestion Probabilities in a Batched Poisson Multirate Loss Model Supporting Elastic and Adaptive Traffic", Annals of Telecommunications, 68 (5), June 2013, pp. 327-344.

[26] I. Moscholios and M. Logothetis, "Call-level blocking of ON-OFF traffic sources in a shared resource environment with batched Poisson arrival processes", Proc. of 19th ITC, Beijing, China, September 2005.

[27] M. Stasiak, M. Glabowski, A.Wisniewski, and P. Zwierzykowski, Modeling and Dimensioning of Mobile Networks, Wiley \& Sons, 2011.

[28] S. Hamalainen, H. Holma and A. Toskala "Capacity evaluation of a cellular CDMA uplink with multiuser detection", Proc. of $4^{\text {th }}$ ISSSTA, September 1996, vol. 1, pp. 339-343.

[29] D. Staehle, K. Leibnitz, K. Heck, B. Schröder, A. Weller, and P. TranGia, "Approximating the othercell interference distribution inhomogeneous UMTS networks", Proc. IEEE VTC 2002, pp. 16401644, 6-9 May 2002.

[30] D. Staehle and A. Mäder, "An analytic approximation of the uplink capacity in a UMTS network with heterogeneous traffic," Proc. 18th ITC, Berlin, pp. 81-91, 31 Aug. - 5 Sept. 2003.

[31] I. Moscholios, G. Kallos, V. Vassilakis and M. Logothetis, "Congestion Probabilities in CDMA-based networks supporting batched Poisson input traffic", Wireless Personal Communications, vol. 79, issue 2, November 2014, pp. 1163-1186.

[32] Simscript III http://www.simscript.com/.(Accessed on June 2015) 